\title{
Experimental Characterization of A Load Sensor Based on Singlemode - Multimode - Singlemode (SMS) Fiber Structure with A Variation of The Number of Bends
}

\author{
Rionda Kuntaraco ${ }^{1}$, Agus M. Hatta ${ }^{1}$, Sekartedjo ${ }^{1}$, Catur A. Prastyanto ${ }^{2}$, and Indrasurya B. Mochtar $^{2}$
}

\begin{abstract}
A load sensor based on singlemode-multimode-singlemode (SMS) fiber structure is presented in this paper. An SMS fiber structure with core diameter of $50 \mu \mathrm{m}$ and length of $30 \mathrm{~cm}$ of the multimode fiber (MMF) section was chosen as a sensor. The measurement principle of the sensor is based on change of SMS fiber structure power response due to the applied load. A fiber bending and load transmitting (FBLT) device is designed to appropiately bend the SMS fiber structure under applied load in order to make the SMS fiber structure work as a load sensor. The applied load can bend the SMS fiber structure and hence affect the multimode interference (MMI) pattern that occured in SMS fiber structure. The number of bends that occured on SMS fiber structure is determined by the pin number of FBLT device. Five variations of pin number at 3, 5, 7, 9 and 11 pins were chosen. The applied load was varied at $0-100 \mathrm{~kg}$. From the experiments, it is found that the relative outpout power response of the sensor gave a specific MMI pattern for each pin number variation. The configuration of 3 pins on FBLT device has the best response as a load sensor with the sensitivity at $1.32 \mu \mathrm{W} / \mathrm{kg}$ and $\mathrm{R} 2$ at 0.9783 with the longest measurement range from $1.82-91.32 \mathrm{~kg}$.
\end{abstract}

Keywords— Load Sensor, Multimode Interference (MMI), Singlemode-Multimode-Singlemode (SMS) Fiber Structure.

\section{INTRODUCTION}

$\mathrm{F}$ iber optic is one of great discovery which has a major effect to public lifes. Beside its role as the backbone of world's data transmission main system, fiber optic is also widely used as a sensor in various measurement system. Fiber optic sensors began to grow rapidly since the early 1970s after the invention of lasers and fiber optics [1]. At the beginning, the applications were focused on military and aerospace field. With the dramatic improvement of fiber optic technology and the fallen of its component prices, fiber optic sensor has been used in numerous application such as for electromagnetic measurement, strain, pressure, displacement, humidity, temperature viscosity and even chemical measurement (lee, 2003)

As a sensor, fiber optic is multipurpose sensor because of its flexibility and has a lot of natural advantages. The natural advantages of fFiber optic sensors such as immune to electromagnetic interference effect, cheap, lightweight, small size, and low power consumption when compared to the conventional sensor [1].

The measurement system using fiber optic sensor utilize the change of light properties which is guided through fiber optic, namely power, wavelength, phase and polarization. This change is caused by signals from the environment such as temperature, humidity, friction, strain, pressure, etc. Even a slightest transformation form signals will change the characteristic of guided ligh in the fiber optic. This is the main factor that cause fiber

${ }^{1}$ Rionda Kuntaraco, Agus M. Hatta, and Sekartedjo are with Departement of Physics Engineering, Faculty of Industrial Technology, Institut Teknologi Sepuluh Nopember, Surabaya, 60111, Indonesia. Email: kuntaracoo@ep.its.ac.id; amhatta@ep.its.ac.id; sekar@ep.its.ac.id.

${ }^{2}$ Catur A. Prastyanto and Indrasurya B. Mochtar are with Departement of Civil Engineering, Faculty of Civil Engineering and Planning, Institut Teknologi Sepuluh Nopember, Surabaya, 60111, Indonesia. E-mail: catur_ap@ep.its.ac.id; indrasurya@ce.its.ac.id. optic sensors have very high sensitivity. Besides that, fiber optic sensor has a high possibility to be applied for multipoints measurement [3].

Nowadays, fiber optic load sensor has been widely studied. One type of fiber optic load sensor is fiber Bragg grating (FBG) load sensor. FBG load sensor has very high sensitivity and low attenuation level [4]. This sensor works based on the principle of wavelength shift due to the occured strain in the FBG which is caused by applied load. It has high resolution of 1 and has measurement range up to $100 \mathrm{~kg}$ [3]. However, in order to work properly, FBG load sensor requires high speed tunable laser and cascade measurement system.

Another type of fiber optic load sensor is using multimode fiber [5]. The multimode fiber load sensor measures the applied load based on microbends principle. This sensor can measure high applied load up to $3000 \mathrm{~kg}$ with the resolution of $30 \mathrm{~kg}$. The sensitivity of this sensor was obtained at $0.303 \mathrm{~mW} / \mathrm{Kg}$. However, with this sensitivity value, a very high power laser source was needed to make this sensor work properly.

A massive development of singlemode-multimodesinglemode (SMS) fiber structure was started lately. An SMS fiber sensor can be an alternative of fiber optic sensor with good prospect. It can be fabricated easily, low cost and high sensitivity [6]. Several application of SMS fiber sensor namely as a optical refractometer [7] bandpass filter [8], strain sensor [9] and so on.

A research to study the application of SMS fiber structure as a load sensor has been examined [10]. It was demonstrated on the study that SMS fiber structure has high sensitivity and good linearity as a load sensor. In this paper, the variation of the number of bends on SMS fiber structure as a load sensor has been examined.

\section{METHOD}

In this research, a particular tool to obtain bend on SMS fiber structure due to applied load is needed. A fiber bending and load transmitting (FBLT) device was 
designed and fabricated to appropriately bend the SMS fiber structure under applied loads in order to make the SMS fiber structure work as a load sensor. The FBLT device has been fabricated in the previous study [10]. The construction of FBLT device that used in this experiment is shown in Figure 1. The spring system on FBLT device is designed to work at $100 \mathrm{~kg}$ of maximum applied load.

A SMS fiber structure with core diameter of $50 \mu \mathrm{m}$ and length of $30 \mathrm{~cm}$ of the multimode fiber (MMF) section was chosen. This SMS fiber structure has the best response in case of sensitivity and linearity [10]. The SMS fiber structure was fabricated using an optical fusion splicer of Fujikura type FSM-505. The SMF-28 with a core diameter of $4.15 \mu \mathrm{m}$ and the graded index MMF of GIF50C (core diameter $50 \mu \mathrm{m}$ ) were chosen. The SMS fiber structure is installed on FBLT device and has a role to sense the applied load. The SMS fiber structure is connected to the laser source with a wavelength of $1550 \mathrm{~nm}$ and the optical power meter. The laser source of EXFO type FPM-300 and the optical power meter of EXFO type FLS-300 were used.

The load test has been conducted to obtain the response of SMS fiber structure as a load sensor. The number of pins that used on FBLT device was varied. Five variations of pin number namely 3, 5, 7, 9 and 11 pins were chosen in this experiment. The scheme of load test using FBLT device for all variations of pin number are shown in Figure $2-6$. The applied load was varied at $0-100 \mathrm{~kg}$.

From the experiment of load test with variations of the pin number, the configuration of pin number that used on FBLT device with the best response. The best response is determined in term of its measurement range, sensitivity and correlation coefficient of every pin configuration.

The load test against time will be carried out for the FBLT device with the best response. There were 2 tests of the load test against time. The first test was done without load. The purpose of this test is to find out the fluctuation of optical power response from SMS fiber structure when no load is applied. The SMS fiber structure was installed on FBLT device without applied load and the relative optical power response was recorded every 5 seconds for 300 seconds. The test was carried out with particular applied load. The load test method was similar with the first test however the applied load will be changed every 30 seconds.

\section{RESULT AND DISCUSSION}

This phenomenon was affected by the self-imaging effect that occurred in the SMS fiber structure. On the self-imaging point, the attenuation that occurred on SMS fiber structure is minimum so it has maximum output power response. A change of self-imaging effect can be caused by the changes of SMS fiber structure dimension and the effect of bend that occurred on SMS fiber structure. The effective refractive index of SMS fiber structure will be distributed asymmetrically due to the bends that occurred on MMF section of SMS fiber structure.

In this experiment, the different number of pins that used on FBLT device gave different number of deflections that occurred on MMF section of SMS fiber structure. The SMS fiber structure is deformed due to the occurred deflection. The density of SMS fiber structure on the deformation points were changed so that the refractive index distribution of SMS fiber structure on the deformation points were also changed. The distance of a self-imaging point with another self-imaging point on the deflection point is changed due to the change of the refractive index distribution

As shown in Figure 7, the more pins used on FBLT device made the period of sinusoidal pattern of the SMS fiber structure relative output power got smaller. This happened because the addition of pin number that used has increased the number of occurred deflections, so that the self-imaging distance has also decreased. Thus the period of maximum and minimum interference was decreased as well.

Based on table 1, it is known that the FBLT device using configuration of 3 pins has the best response in term of its measurement range, sensitivity and correlation coefficient. This configuration gave the widest load measurement range. It also has single monotonic response for all applied load. The increasing of applied load made the relative output power of SMS fiber structure decreased for the whole applied load range. It has good sensitivity at $1.32 \mu \mathrm{W} / \mathrm{kg}$ and correlation coefficient (R2) at 0.9783 , highest among all configurations.

\section{CONCLUSION AND FUTURE WORK}

A load effect based on SMS fiber structure with a variation of the number of bends has been presented. The SMS fiber structure with core diameter of $50 \mu \mathrm{m}$ and length of $30 \mathrm{~cm}$ of the MMF section has been chosen to be installed on FBLT device. It is demonstrated that the number of bends has significant effect against the relative output power that occured on the SMS fiber structure. It is also found that the relative output power response of the sensor gave a specific MMI pattern for each pin number variation. The SMS fiber structure with configuration of 3 pins on FBLT device has the best response as a load sensor with the sensitivity at 1.32 $\mu \mathrm{W} / \mathrm{kg}$ and R2 at 0.9783 with the longest measurement range from 1.82 - $91.32 \mathrm{~kg}$. Further research to develop SMS fiber structure load sensor for measuring dynamic load will be carried out.

\section{ACKNOWLEDGEMENT}

The authors acknowledge General Directorate of Higher Education (DIKTI), Indonesia ministry of education and culture for financial support by PENPRINAS MP3EI contract no. 1221.09/IT2.7/PN.01/ 2012. The author's gratitude also go to PT Telkom Indonesia for lending us several equipments that used in this research.

\section{REFERENCES}

[1]. F.T.S. Yu and S. Yin, "Fiber Optic Sensors”, 1st edition, Marcel Dekker Inc, New York, 2002.

[2]. B. Lee, "Review of present status of optical fibre sensors", Optical Fibre Technology, vol. 9, pp. 57 - 79, 2003.

[3]. D. Tosi, M. Olivero, A. Vallan, and G. Perrone, "Weigh-inmotion through fibre Bragg-grating optical sensors”, Electronics Letters, vol. 46, pp. 17, 2010. 
[4]. F. Urban, J. Kadelc, R. Vlach, and R. Kuchta, "Design of a Pressure Sensor Based on Optical Fiber Bragg Grating Lateral Deformation”, Sensors, vol. 10, pp. 11212 - 11225, 2010.

[5]. M. Bin and Z. Xinguo, 2010, "Study of Vehicle Weigh-InMotion System Based on Fiber Optic Microbend Sensor", presented at the International Conference on Intelligent Computation Technology and Automation, IEEE, Changsa, China, 2010

[6]. L. B. Soldano, and E. C. M. Pennings, "Optical Multimode Interference Devices Based on Self-Imaging : Principles and Applications”, Journal of Lightwave Technology, vol. 13, pp. $515-627,1995$.

[7]. R.X. Gao, W.J. Liu, Y.Y. Wang, Q. Wang, Q. Zhao and S.L. Qu, "Design and Fabrication of SMS Fiber Refraction". Sensor and Actuator, vol. 79, pp. 5-9, 2012.

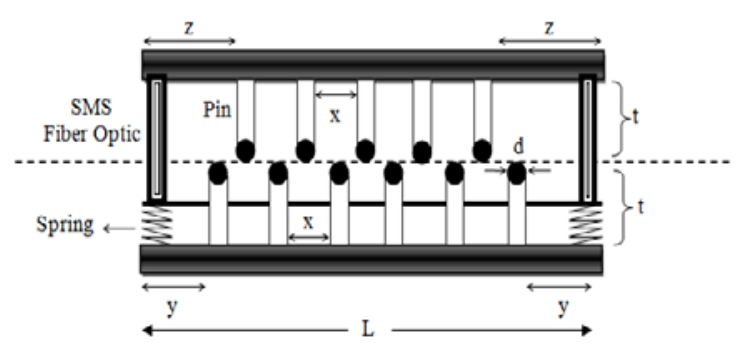

Figure 1. The construction of FBLT device [10]

$$
\text { Note: } \begin{array}{ll}
\mathrm{L}=24 \mathrm{~cm} & \mathrm{x}=2 \mathrm{~cm} \\
\mathrm{t}=5 \mathrm{~cm} & \mathrm{y}=4 \mathrm{~cm} \\
\mathrm{z}=5.5 \mathrm{~cm} & \mathrm{~d}=1 \mathrm{~cm}
\end{array}
$$

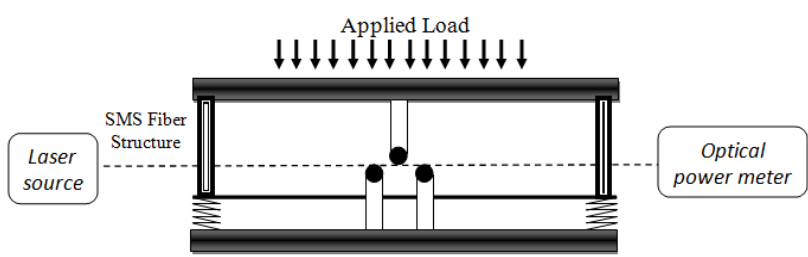

Figure 2. Scheme of FBLT device using 3 pins

Applied Load

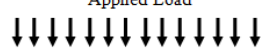

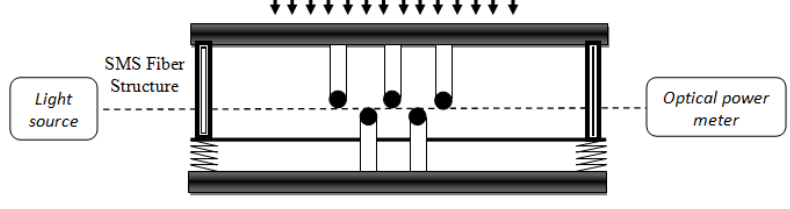

Figure 3. Scheme of FBLT device using 5 pins
[8]. Q. Wang, G. Farrell and W. Yan, "Investigation on SinglemodeMultimode-Singlemode Fiber Structure". IEEE Trans. Journal of Lightwave Technology, vol. 26, pp. 512 - 519, 2008

[9]. A. M. Hatta, Y. Semenova, Q. Wu and G. Farrell, "Strain sensor based on a pair of singlemode-multimode-singlemode fiber structures in a ratiometric power measurement scheme,” Applied Optics, vol. 49, pp. 536-541, 2010.

[10]. R. B. Kuntaraco, A. M. Hatta, Sekartedjo, C. A. Prastyanto dan I. B. Mohctar, "Characterization of Singlemode-MultimodeSinglemode (SMS) Fiber Optic as a Load Sensor Based on Macrobending Mechanism”, presented at the Seminar Pascasarjana ITS, Surabaya, Indonesia, 2013.

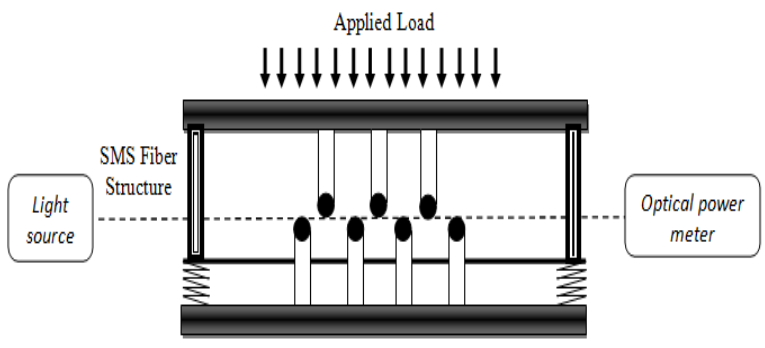

Figure 4. Scheme of FBLT device using 7 pins

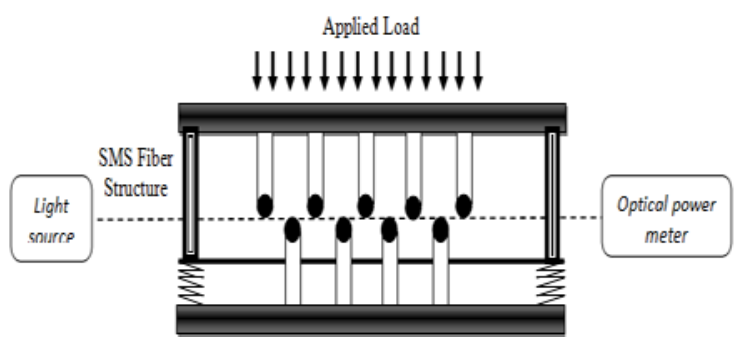

Figure 5. Scheme of FBLT device using 9 pins

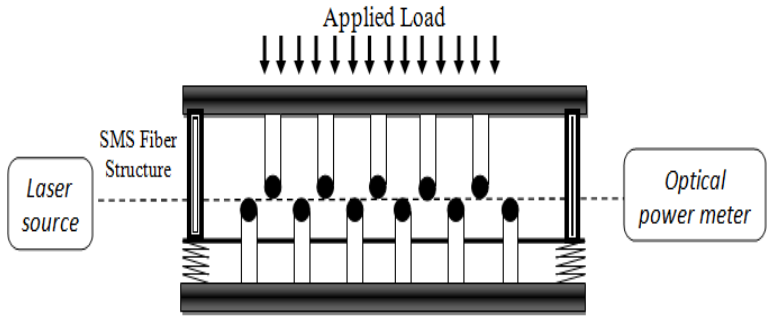

Figure 6 Scheme of FBLT device using 11 pins

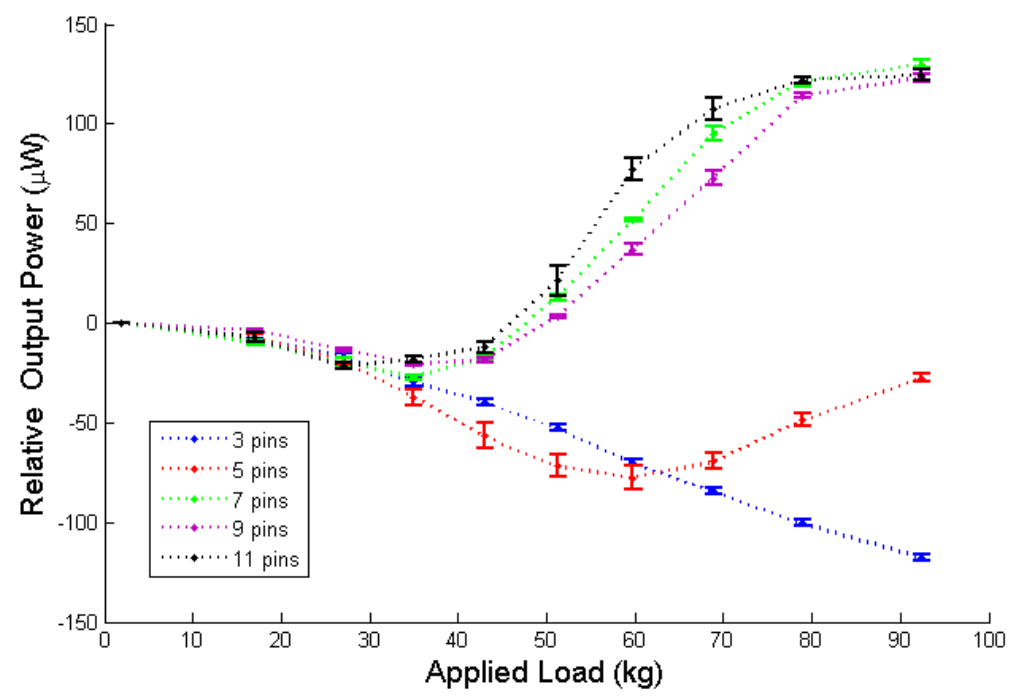

Figure 7. The result of applied load effect on the SMS fiber structure for all variations of pin used 
TABLE 1.

APPLICABLE MEASUREMENT RANGE, SENSITIVITY AND CORRELATION COEFFICIENT OF SMS FIBER STRUCTURE LOAD SENSOR FOR ALL VARIATIONS OF PIN NUMBER OF FBLT DEVICE

\begin{tabular}{cccc} 
Number of pin & $\begin{array}{c}\text { Applicable Measurement } \\
\text { Range }(\mathrm{kg})\end{array}$ & $\begin{array}{c}\text { Sensitivity } \\
(\mu \mathrm{W} / \mathrm{kg})\end{array}$ & $\begin{array}{c}\text { Correlation Coefficient } \\
\left(\mathrm{R}^{2}\right)\end{array}$ \\
\hline 3 & $1,82-91,32$ & 1,32 & 0,9783 \\
5 & $1,82-59,78$ & 1,34 & 0,9514 \\
7 & $34,99-91,32$ & 2,79 & 0,9583 \\
9 & $34,99-91,32$ & 2,55 & 0,9619 \\
11 & $26,99-91,32$ & 2,27 & 0,9171 \\
\hline \hline
\end{tabular}

